Research Article

\title{
Identification of 24 Unknown Substances (NIAS/IAS) from Food Contact Polycarbonate by LC-Orbitrap Tribrid HRMS-DDMS ${ }^{3}$ : Safety Assessment
}

\author{
Vicent Yusà $\left(\mathbb{D},{ }^{1,2}\right.$ Antonio López, ${ }^{1}$ Pablo Dualde, ${ }^{1}$ Olga Pardo, ${ }^{1}$ Igor Fochi, ${ }^{3}$ \\ Pablo Miralles, ${ }^{1}$ and Clara Coscollá ${ }^{1}$ \\ ${ }^{1}$ Foundation for the Promotion of Health and Biomedical Research in the Valencian Region, FISABIO-Public Health, \\ Av. Catalunya, 21, Valencia 46020, Spain \\ ${ }^{2}$ Public Health Laboratory of Valencia, 21, Av. Catalunya, Valencia 46020, Spain \\ ${ }^{3}$ Thermo Fisher, Rodano, MI, Italy \\ Correspondence should be addressed to Vicent Yusà; yusa_vic@gva.es
}

Received 18 November 2020; Revised 17 February 2021; Accepted 25 February 2021; Published 27 March 2021

Academic Editor: Chanbasha Basheer

Copyright (C) 2021 Vicent Yusà et al. This is an open access article distributed under the Creative Commons Attribution License, which permits unrestricted use, distribution, and reproduction in any medium, provided the original work is properly cited.

Twenty-four substances, mainly NIAS, have been tentatively identified in food contact polycarbonate through the application a new, fast, and automated analytical strategy for the investigation of unknowns in food contact materials. Most of the identified compounds were plasticizers, slip agents, antioxidants, and ultraviolet stabilizers and fragrances, and the majority of them have not been previously identified in PC food contact materials. The workflow setup includes an intelligent data acquisition applied using LC-Orbitrap Tribrid-HRMS $\left(\mathrm{MS}^{3}\right)$, with an automated data processing using Compound Discoverer ${ }^{\mathrm{TM}}$. To obtain a high confidence identification of unknown substances, a very strict criterion has been established, which comprises exact mass, isotopic profile, $\mathrm{MS}^{2}$ match, retention time, and $\mathrm{MS}^{3}$ match. To check for the safety of the migration from the food contact polycarbonate, a risk assessment was achieved using the threshold of the toxicological concern (TTC) approach. Except for the slip agent hexadecanamide, the compounds tentatively identified do not represent a risk.

\section{Introduction}

Plastics for food contact applications must be safe. In Europe, plastic articles may only be placed on the market as food contact materials (FCM) if they are manufactured with substances (monomers, additives, and aids) included in the list incorporated into the Regulation 10/2011 [1] (intentionally added substances, IAS). However, during their manufacturing and use, some impurities and reaction or degradation products can be formed (nonintentionally added substances, NIAS). The potential risk of NIAS should be considered for authorization $[2,3]$ and for the declaration of compliance at the marketing stages [4].

Between the different thermoplastic materials used as FCM, polycarbonate (PC) is commonly employed for tableware and containers exposed to hot filling or heating after filling, such as plates and bowls, that could be used repeatedly. These PC containers can also be used for reheating food in a microwave oven [5]. Many ambient factors such as temperature, humidity, light together with the repeated uses, and washing cycles can damage the stability of the material and increase the migration of substances to the food [6]. The main concern about degradation of PC food containers is the release of bisphenol A (BPA), a component monomer of PC that is currently of toxicological concern $[7,8]$.

To assess the safety of food contact plastic materials, appropriate analytical methods are required [9]. Apart from the target analysis, mainly for detection of IAS or specific contaminants, the analysis of NIAS is mandatory to avoid any potential health risk to the consumer [2] and should be performed in accordance with the Regulations 1935/2004 [4] and 10/2011 [1]. The identification of NIAS is challenging 
and requires a non-target analytical approach $[10,11]$ using LC-HRMS (Q-Orbitrap/Q-TOF), performed using two main modes, data-dependent acquisition (DDA) and dataindependent acquisition (DIA) [12].

Bignardi et al. [13] employed a target and untargeted method, using LC-HRMS-Orbitrap working in the datadependent acquisition mode (DDA), for characterization of PC food contact plastic. In the target approach, several plastic additives were identified. With the untargeted analysis, some colorants and several oligomers derived from the chain breakage were identified, following a laborious and time-consuming process. In a recent study, the same research team [14] applied the target method with UHPLCHRMS to determine the migration from PC tableware objects, detecting oligomers from the PC chain and traces of colouring agents.

To perform an exhaustive characterization of NIAs, an extensive acquisition (DDA including Full Scan and $\mathrm{MS}^{\mathrm{n}}$ ) and identification of the unknown substances is necessary. This is challenging because of the large number of features present both in the samples and the background (blanks and/or matrix). In order to automate these procedures, new acquisition tools are developed for background removing, exhaustive precursor selection (inclusion list), and creation of dynamic and real-time exclusion list [15]. Likewise, an automated library search has been improved through the data processing tool Compound Discoverer ${ }^{\mathrm{TM}}$ [16].

For the identified NIAS, a risk assessment is necessary to ensure the safe usage of the PC containers. One practical option is to use the threshold of the toxicological concern (TTC) approach that estimates the theoretical toxicity of compounds linked to their molecular structure, and a threshold value is provided below which there is a very low probability of adverse health effects $[17,18]$.

In this study, a new approach for the determination of unknown substances in PC bowls used for food container was investigated. We evaluated the capacity for exhaustive spectral data acquisition of an innovative intelligent acquisition mode (Acquire $\mathrm{X}^{\mathrm{TM}}$ ) using $\mathrm{DD}-\mathrm{MS}^{3}$. In the second phase, the automatic search on compound libraries and spectral databases was employed to putative identification of unknown compounds. Finally, we assessed the risk of the detected migrating substances.

\section{Materials and Methods}

2.1. Chemicals and Samples. Chemical standards of terfenadine and Val-Try-Val were from Sigma Life Science (St Louis, MO, USA), triallyl phosphite was from Alfa Aesar Thermo Fisher (Kandel, Germany), and sulfaguanidine, sulfadimethoxine, reserpine, caffeine, and acetaminophen were obtained from Sigma Aldrich (St Louis, MO, USA). Solutions of $10 \mathrm{ng} / \mathrm{mL}$ of these chemicals were prepared in $\mathrm{H}_{2} \mathrm{O}: \mathrm{MeOH}(70: 30, \mathrm{v} / \mathrm{v})$ and used as internal standards.

Chemical standards of bis (2-ethylhexyl) adipate, camphor, dibutyl sebacate, dibutyl phthalate, erucamide, 2-(2'hydroxy-5'-methylphenyl) benzotriazole (benazol P), methyl dihydrojasmonate, oleamide, and tributyl acetyl citrate (Citroflex-A4), all from Sigma Life Science (St Louis,
MO, USA), and diethyl phthalate, dibutyl adipate, and tris(2,4-ditert-butylphenyl) phosphite (Irgafos 168), from LGC Standards (Bury, United Kingdom), were used to quantify and confirm the identity of the tentatively identified substances.

We analysed polycarbonate bowls that, according with the declaration of conformity, were used in repeated contact with miscellaneous foods such as soups and broths, during a maximum time contact of 2 hours and a temperature between $85^{\circ} \mathrm{C}$ and $89^{\circ} \mathrm{C}$ and prepared to endure until 2000 washing cycles.

2.2. Migration Test and Sample Preparation. Migration tests were achieved in agreement with the Regulation (EU) No. $10 / 2011$ [1]. We used the food simulant C (20\% of ethanol in water $(\mathrm{v} / \mathrm{v}))$. The migration test was performed during 2 hours at $100^{\circ} \mathrm{C}$. We introduced around $200 \mathrm{~mL}$ of simulant into the PC rectangular bowl $(10 \mathrm{~cm} \times 5.5 \mathrm{~cm} \times 3 \mathrm{~cm})$ and keep it in the incubator. According with Regulation (EU) No. 10/2011, the migration test was repeated three times. Taking into account this Regulation, $6 \mathrm{dm}^{2}$ is equal to $1 \mathrm{Kg}$ of food.

2.3. LC-HRAMS Analysis. We used a Thermo Ultimate 3000 UHPLC system, with a column Hypersil Gold $100 \mathrm{~mm} \times 2.1 \mathrm{~mm} \times 1.9 \mu \mathrm{m}$ (Thermo Scientific). The chromatographic system was linked with an Orbitrap ID-X Tribrid mass spectrometer (Thermo Fisher Scientific, USA). A volume of $5 \mu \mathrm{L}$ was injected, and the flow rate was $300 \mu \mathrm{L} / \mathrm{min}$. The chromatography started with $90 \%$ phase A (water) and $10 \% \mathrm{~B}$ (methanol). The linear gradient used was 0-18 min, $70 \% \mathrm{~B}$; $18-21.5 \mathrm{~min}, 98 \% \mathrm{~B}$; sustained at $98 \% \mathrm{~B}$ from $21.5 \mathrm{~min}$ to $25 \mathrm{~min}$; decrease to $10 \% \mathrm{~B}$, from $25 \mathrm{~min}$ to $26 \mathrm{~min}$; and maintained in this initial conditions until $30 \mathrm{~min}$.

Both positive and negative ionizations were employed. The H-ESI parameters were electrospray voltage of $3.5 \mathrm{kV}$ positive (3.2 Kw negative), sheath gas of 25 arbitrary units (a.u), and auxiliary gas of 5 a.u. The ion transfer tube worked at $270^{\circ} \mathrm{C}$ and the vaporizer temperature at $180^{\circ} \mathrm{C}$. A resolving power of 120.000 FWHM was used in full scan $(100-900 \mathrm{~m} / \mathrm{z})$. The $\mathrm{MS}^{2}$ was acquired at $15.000 \mathrm{FWHM}$, with a precursor mass range of $125-900 \mathrm{~m} / \mathrm{z}$. The $\mathrm{MS}^{3}$ precursor was isolated in the quadrupole mass filter $(0.4 \mathrm{Da})$, fragmented in the HCD cell and detected in the ion trap $(\mathrm{FWHM} \leq 0.3)$.

2.4. Acquisition and Data Processing. For unknowns analysis, an intelligent data approach (AcquireX ${ }^{\odot}$, ThermoFisher Scientific) was employed. The details of this approach were described previously [19]. The acquisition workflows for Deep Scan (DS) and Interative Processing Exclusion (IPE) modes in DD-MS ${ }^{3}$ are shown in Figures SI-1-2.

For compound identification, the raw files acquired were processed using Compound Discoverer ${ }^{\mathrm{TM}} 3.1$ (CD) (Thermo Fisher Scientific). The CD workflow is shown in Figure SI-3, and each node is defined in Table SI-1. The CD provides a 


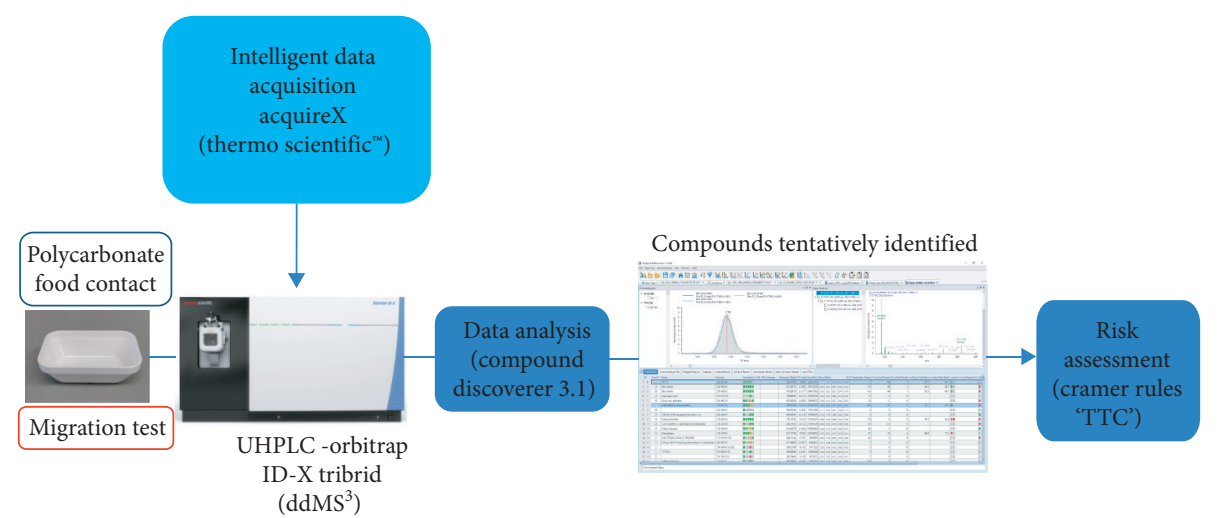

Figure 1: Scheme of the different stages of the presented study.

wide list of annotated compounds (several hundreds). However, the majority of them do not present enough analytical evidence for tentative identification. To consider a compound tentatively identified with a high degree of confidence, we used the criteria previously described by [19]. In short, exact mass is with a $\Delta$ mass $<0.5 \mathrm{ppm}$; isotopic profile $(\mathrm{SFit} \%)>70 \%$; $\mathrm{MS}^{2}$ match (experimental vs. library (mzCloud) spectra) $>70 \%$; retention time consistent with their $\log \mathrm{P}$.

2.5. Safety Assessment. For safety assessment of the identified NIAS, we use the threshold of the toxicological concern (TTC) approach [2]. This was performed using the Toxtree software (http://toxtree.sf.net/predict) developed by Idea Consult Ltd., commissioned by JRC Computational Toxicology and Modelling (https://apps.ideaconsult.net/data/ui/ toxtree). The software estimates the TTC following Cramer rules, and for each of the three Cramer classes, a tolerable daily intake (TDI, mg/person/day) is assigned. Class I, the least toxic class, has a TDI $=1.8 \mathrm{mg} /$ person/day, Cramer class II (intermediate) has a TDI $=0.54 \mathrm{mg} /$ person $/$ day, and Cramer class III (most toxic) has a TDI $=0.09 \mathrm{mg} /$ person/ day.

The estimated daily intake (EDI) was calculated based on the default assumption in Europe that an adult person consumes $1 \mathrm{Kg}$ of food packaged in a $\mathrm{dm}^{3}$, using the following formula [20].

EDI $(\mathrm{mg} /$ person $x$ day $)=$ migration $(\mathrm{mg} / \mathrm{kg}) \times 1 \mathrm{~kg}($ food intake per person and day).

Quantification was performed using pure standards (Section 2.1). When standards were not available, a semiquantitative estimation of the migration $(\mathrm{mg} / \mathrm{Kg})$ was performed with the average response factor of seven internal standards [21] (Table SI-2).

Figure 1 shows the diagram of the overall analytical procedure.

\section{Results and Discussion}

3.1. Identification of Unknowns. Out of 727 annotated features with CD, only 24 meet the metrics established for tentative identification of compounds (Table 1). In order to achieve a high degree of identification confidence, very demanding criteria were followed (Section 2.5). All of them present a mass error $(\Delta \mathrm{M})$ in general lower than $0.5 \mathrm{ppm}$. Equally, the isotopic patterns present, in general, a SFit\% $>70 \%$. Likewise, their $\mathrm{MS}^{2}$ spectra match (>70\%) with the spectra in the mzCloud library (Table 1). As an orthogonal criteria for increasing the identification confidence, the experimental retention time $\left(\mathrm{Rt}_{\mathrm{ex}}\right)$ was compared with the theoretical one $\left(\mathrm{Rt}_{\mathrm{th}}\right)$. To estimate the theoretical retention time, we build a curve that relates the $\log \mathrm{P}$ with the Rt of 107 substances of a wide range of polarities (Figure SI-4). Using this curve, we estimated the $\mathrm{Rt}_{\mathrm{th}}$ of the identified substances. In general, the $\mathrm{Rt}_{\mathrm{ex}}$ (min) fits well with the $\mathrm{Rt}_{\text {th }}$ (Table 1).

For identification of those substances that do not meet the established criteria (only the molecular formula was provided by $\mathrm{CD}$ ), a more in-deep and time-consuming manual examination is required, using in silico fragmentation tools and bibliographic examination [12].

In previous published studies for non-target screening applied to FCM, acquisitions using the data-dependent approach (DDA) with Q-Orbitrap and data-independent approach (DIA) with Q-TOF-MS instruments have been used [12]. The identification strategy is performed manually utilizing lists of probable candidates (only formula available), spectral libraries (MS/MS ${ }^{2}$ spectra), and bibliographical search. The main drawback of this strategy is that it is labour-intensive, and only a few compounds are tentatively identified. Regarding PC food materials, only a few substances migrating to simulants have been described. As mentioned previously, Bignardi et al. [13] and Bignardi et al. [14] performed a targeted and untargeted suspect screening to identify substances migrating from food contact polycarbonate using DDA-MS ${ }^{2}$ acquisition. The identification of compounds was carried out using a list of additives (target) and through the careful observation of the full scan chromatograms, the evaluation of the isotopic pattern, and the investigation in available databases. In the untargeted analysis, they claim the identification of several organic colorants and polycarbonate degradation products, but no $\mathrm{MS}^{2}$ spectra were used for structural identification. Another study [6] investigated, using a target method with LCHRMS, the presence of the PC monomer bisphenol A (BPA) and several additives in polycarbonate tableware. BPA was 


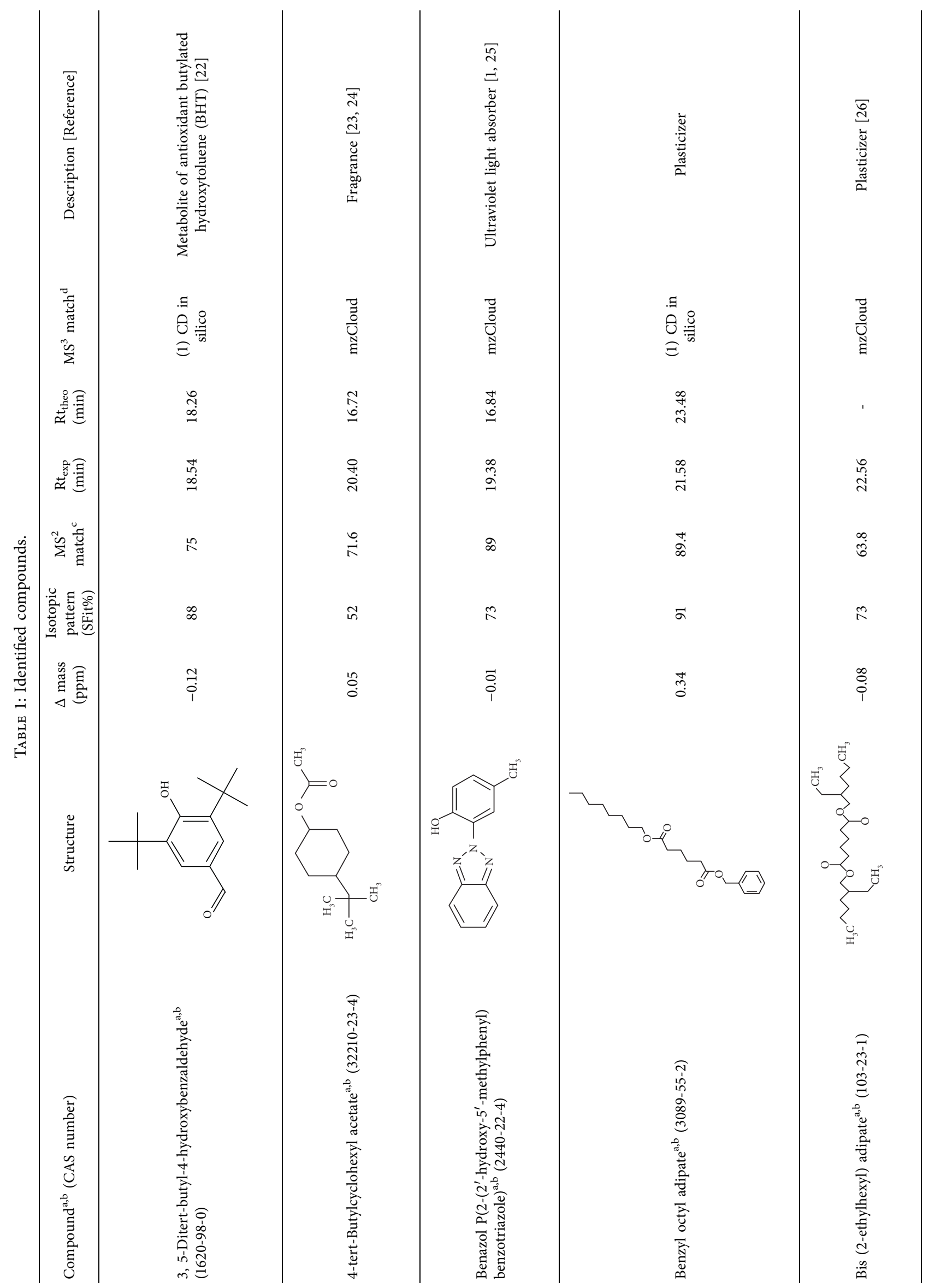














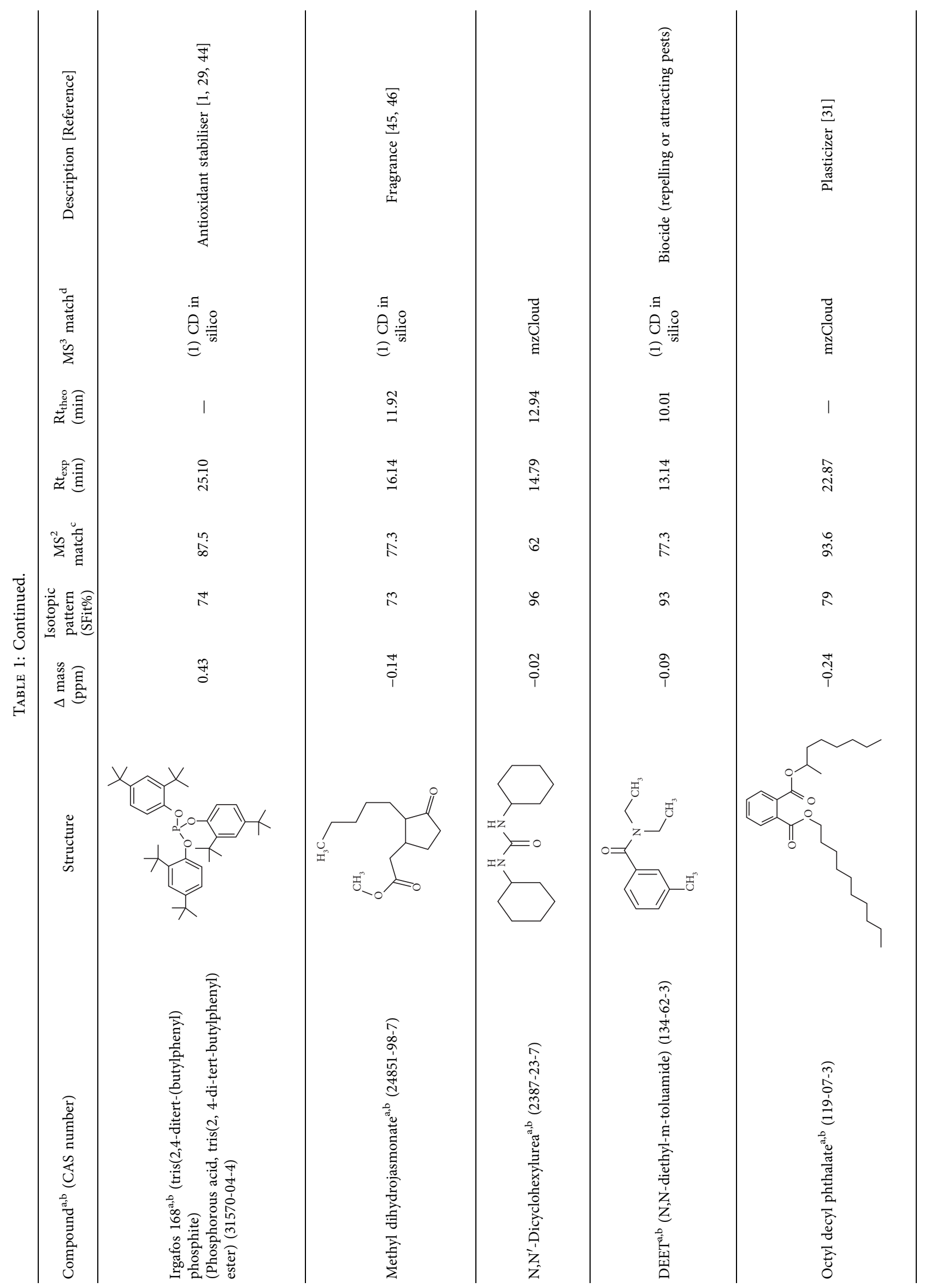




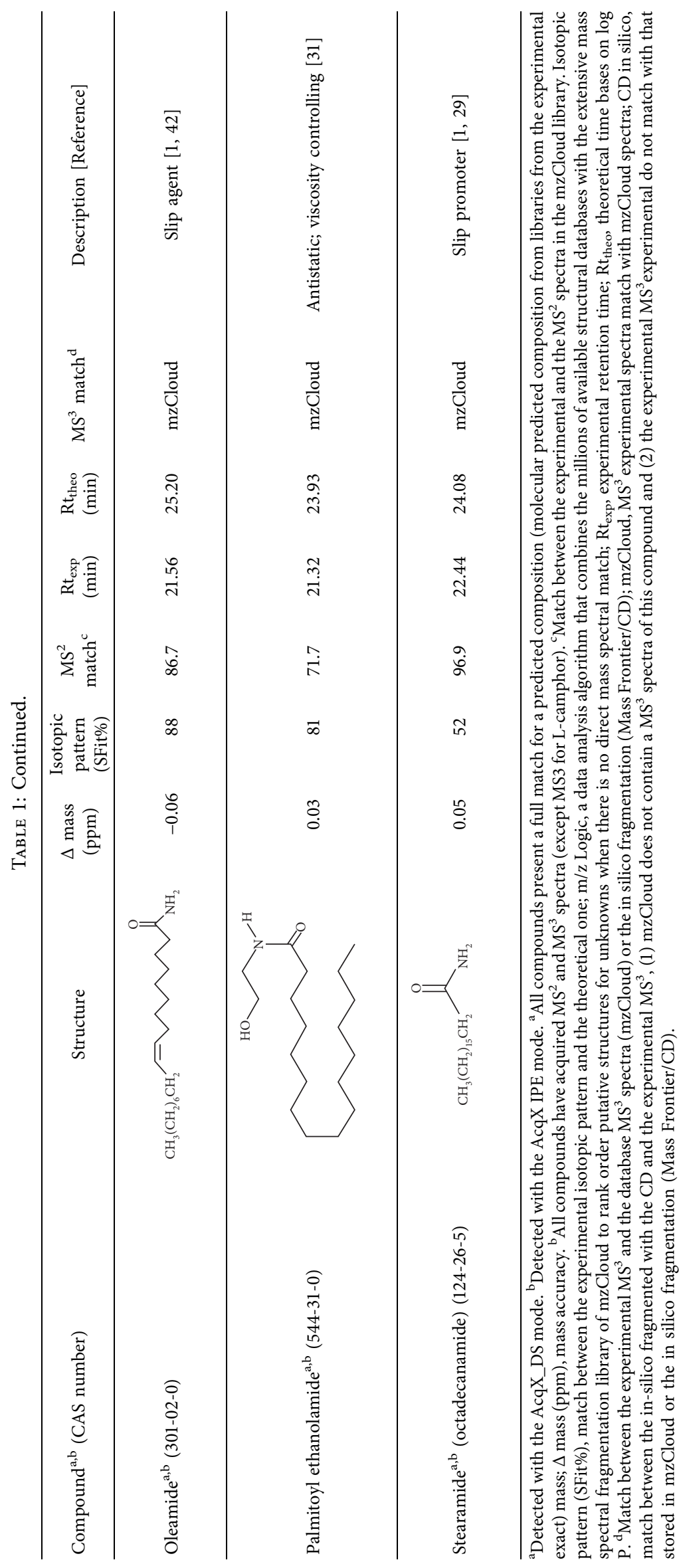




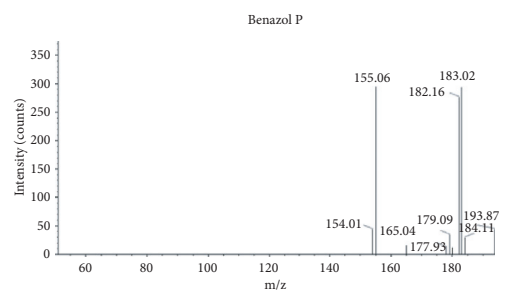

(a)

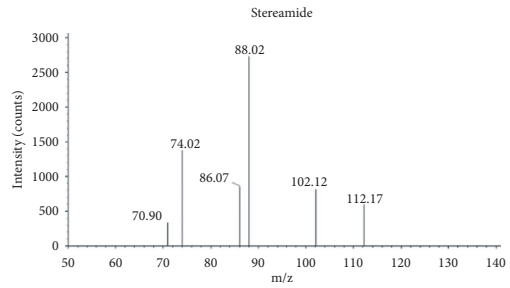

(c)

Figure 2: (a) Experimental $\mathrm{MS}^{3}$ spectra and (b) mzCloud $\mathrm{MS}^{3}$ spectra for benazol $\mathrm{P}$ and stearamide.

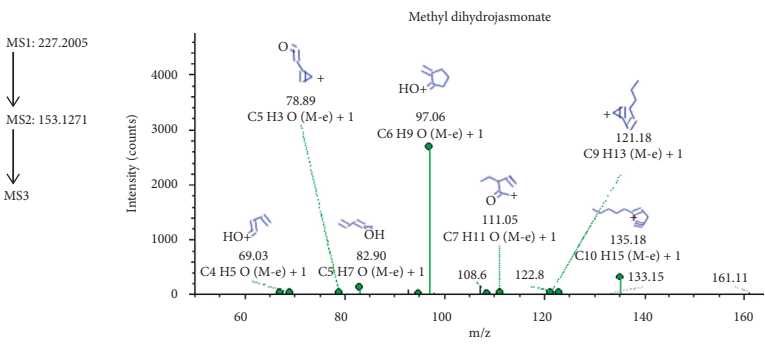

(a)
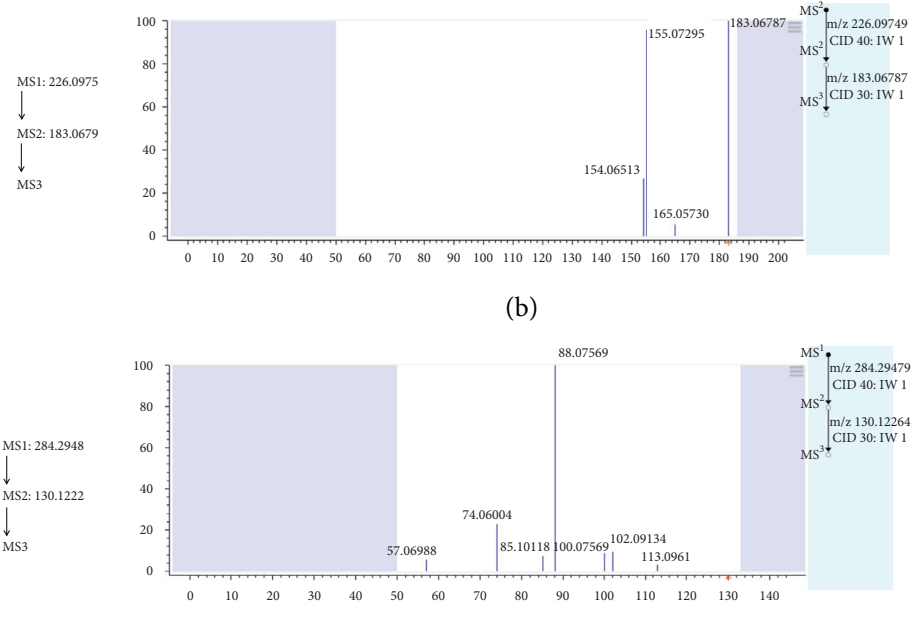

(d)

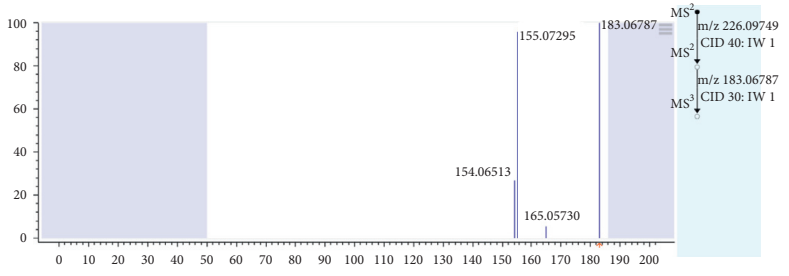

(b)


(b)

FIGURE 3: Experimental $\mathrm{MS}^{3}$ spectra for methyl dihydrojasmonate and benzyl octyl adipate. The structures highlight the experimental MS ${ }^{3}$ signals which matched with the in silico fragments obtained using Compound Discoverer.

determined in all samples (in the range of $0.5-6 \mu \mathrm{g} / \mathrm{Kg}$ ), and the UV absorbers Cyasorb UV 5411, Tinuvin 327, and Tinuvin 324 and the whitening Uvitex OB were found in some samples (from $7 \mathrm{ng} / \mathrm{Kg}$ to $4460 \mathrm{ng} / \mathrm{Kg}$ ). The target analysis, in general, permits achieve lower LOQ.

3.1.1. $M S^{3}$ Spectra. The automated identification achieved by the combination of the intelligent data acquisition $\left(\right.$ AcqX-ddMS ${ }^{3}$ ) combined with the data processing using CD does not use the $\mathrm{MS}^{3}$ spectra. A higher level of confidence could provide the $\mathrm{MS}^{3}$ spectra. As can be seen in Table 1 and Table SI-3, all compounds identified have their $\mathrm{MS}^{3}$ acquired spectrum, except for D,L-camphor. However, it is necessary to compare the experimental and the library (mzCloud) $\mathrm{MS}^{3}$ spectra manually. For those compounds that do not have $\mathrm{MS}^{3}$ spectra in mzCloud, we fragmented them in silico using $\mathrm{CD}$ and Mass Frontier 8.0. [32].

Ten of the identified compounds had $\mathrm{MS}^{3}$ spectra that matched with that stored in spectrum library. Figure 2 shows, as an example, the spectra of benazol $\mathrm{P}$ and stearamide. In both $\mathrm{MS}^{3}$ spectra, the experimental parent ions $(\mathrm{m} / \mathrm{z} 183.0679 ; \mathrm{m} / \mathrm{z} 130.1222$, respectively) and the main fragments (m/z 155.06 and $\mathrm{m} / \mathrm{z}$ 88.02, respectively) match with the library spectra. Must be taken into account that $\mathrm{MS}^{3}$ was acquired in low resolution. On the other hand, there are eleven substances in which experimental MS ${ }^{3}$ spectra match with the in silico fragmentation performed with the $\mathrm{CD}$. As an example, Figure 3 depicts the experimental spectra of the methyl dihydrojasmonate and benzyl octyl adipate and the in silico fragments assigned to each $\mathrm{m} / \mathrm{z}$.

Using the criteria proposed by Schymansky et al. [33], the tentatively identified compounds present a high level of identification confidence (level 2a: unambiguous matching with library data). The other annotated compounds (not discussed here), for which only the molecular formula $o$ exact mass is known, only achieve levels 4-5 (low levels of identification confidence).

We have confirmed the identity of 12 out of 24 tentatively identified substances using standards (Section 2.1). The rest of substances were not available in our lab.

3.1.2. Description of Identified Compounds. In order to explain the presence in the polycarbonate plastic material of the identified compounds, we search for their nature and technological uses. Table 1 shows the functions of these compounds. Many of them are additives, mainly plasticizers and slip agents used in plastics. Additives are introduced 


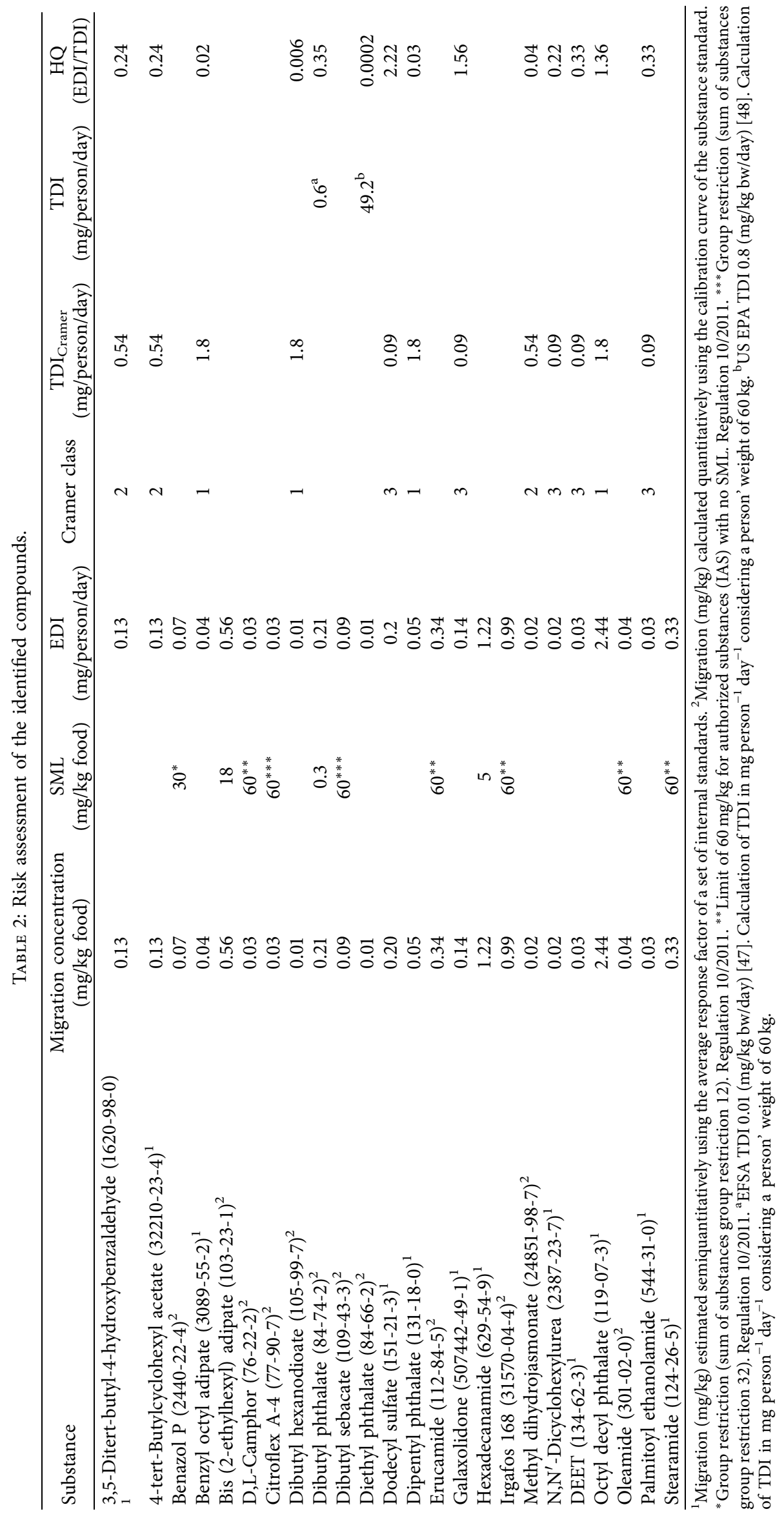


into plastic to enhance the quality of the attributes required for their appropriate use. They improve their physical (mechanical, thermal, and durability) and chemical properties and aid with processing of polymers [34].

Plasticizers are additives used to provide flexibility and improve their process ability, and between them, orthophthalates are the most used plasticizers [35]. The typical amount range $10-70 \%(\mathrm{w} / \mathrm{w})$. Among the identified substances are included four phthalates (DEP, DBP, DPP, and ODP). Both DBP and DPP are included in the "Authorisation List" of Annex XIV of REACH [36] for their toxic effects for reproduction. However, DBP are listed in the Union List of the Regulation 10/2011 to be used as plasticizer in repeated use materials and articles contacting nonfatty foods with a specific migration limit (SML) of $0.3 \mathrm{mg} / \mathrm{Kg}$ (we have detected $1.34 \mathrm{mg} / \mathrm{Kg}$ ). Contrarily, neither DEP nor DPP and ODP are in the Union List. Two other plasticizers such as Citroflex A-4 and bis (2-ethylhexyl) adipate are also included Union List of authorized substances (R. 10/2011).

Another group of identified substances is the slip agents, which reduce friction during and after plastic manufacturing and are used in the range of $0.05-3 \%(\mathrm{w} / \mathrm{w})$ [37]. Oleamide, erucamide, stearamide, and hexadecanamide are authorized substances to be used as additives in plastics (IAS).

Antioxidants and ultraviolet stabilizers are widely used additives in plastics, usually in the range of $0.05-3 \%$ to enhance the lifetime of polymeric material, avoiding the oxidation process by oxygen or UV light [34, 37]. Benazol P is a benzotriazole ultraviolet (UV) light absorber that provides UV protection to a wide variety of polymers, including polycarbonates. It is an authorized substance included in the Union List, with a total specific migration limit (SML-T) of $30 \mathrm{mg} / \mathrm{Kg}$ for the restriction group of the benzotriazole-type UV stabilizers (three substances). Irgafos $168^{\odot}$ is a phosphite antioxidant that provides polymers protection against thermooxidative degradation and is used in small amounts, usually between $0.004 \%$ and $0.5 \%$ [29]. It is included in the Union List (IAS).

We have also identified four odorants, which are added into plastics to add desirable fragrance or to mask any undesirable odor. 4-tert-Butylcyclohexyl acetate and methyl dihydrojasmonate are included in the EU cosmetics database [24] as perfumes. D,L-camphor is used as flavouring substance in foods and for perfuming industrial products [38]. On the other hand, galaxolidone is a metabolite of galaxolide, a polycyclic musk widely used as a fragrance in personal care and consumer products.

To our knowledge, these substances have not been previously determined in the polycarbonate food contact material. In a posterior target analysis using a target LC-MS/ MS method, we determined BPA with concentrations ranging from $0.002 \mathrm{mg} / \mathrm{Kg}$ to $0.008 \mathrm{mg} / \mathrm{Kg}$ (details not provided). These low levels are difficult to detect with the wide scope of the untargeted methods.

3.2. Safety Assessment. For FCM, it is mandatory to guarantee their safety regarding the IAS and NIAS migration. For IAS, the migration concentration should be compared with the specific migration level (SML), established in the Regulation 10/2011. For those authorized substances with no SML, we have used the limit of $60 \mathrm{mg} / \mathrm{Kg}$ food (limit for overall migration).

For NIAS with toxicological reference values (TDI), we use this as a threshold (Table 2). For the rest of identified compounds, we applied the TTC approach described previously (Section 2.5).

The IAS benazol P, bis (2-ethylhexyl) Citroflex A-4, and hexadecanamide present a migration concentration lower than the established SML; therefore, their exposures are not of concern (Table 2). None of the authorized substances without SML exceeds $60 \mathrm{mg} / \mathrm{Kg}$ food. The rest of the identified substances show a $\mathrm{HQ}<1$; consequently, their migration does not represent a risk. The HQ is not a regulated limit but a risk metric.

More in-deep investigation is needed to uncover the identity of all annotated but not identified substances and to perform a risk assessment.

\section{Conclusions}

A new and automated analytical strategy for identification of unknown substances in food contact materials was applied to food contact polycarbonate and permitted the identification of 24 substances, mainly plasticizers, slip agents, and antioxidants. The majority of these substances have not been previously identified in PC food contact materials.

The use of the intelligent data acquisition $\left(\right.$ Acquire $^{\odot}$ ) in both modes (DE and IPE) has permitted a fast and exhaustive data acquisition, and the use of DDA-MS ${ }^{3}$ provides profuse structural information that increase the identification confidence.

The implementation of an automated workflow for database searching is essential to avoid labour intensive and time-consuming explorations. The use of a specific $\mathrm{CD}$ workflow has permitted a very fast search in different databases, which could be improved increasing the database content with more substances.

We have defined very rigorous criteria for identification of the annotated substances, in order to provide to the analytical strategy with a high level of identification confidence. The use of $\mathrm{MS}^{3}$ provides more structural information that increases this confidence. However, some general accepted guidelines for NIAS identification are necessary.

4.1. Study Limitations. For several identified substances, a semiquantitative migration $(\mathrm{mg} / \mathrm{kg})$ was estimated using the average response of seven internal standards not chemically related with the identified substances. This, obviously, is a rough approximation for risk assessment that could be improved using the standards of the identified substances.

\section{Data Availability}

The data used to support the findings of this study are available from the corresponding author upon request. 


\section{Conflicts of Interest}

The authors declare that they have no conflicts of interest.

\section{Acknowledgments}

The study was performed using analytical instruments financed by the European Commission through the European Regional Development Funds (ERDF) Operational Programme of the Valencia Region (2014-2020). Pablo Dualde acknowledges his "Ayudas para la contratación PTA" (PTA2018-016320-I) from "Ministerio de Ciencia e Innovación" (Spain).

\section{Supplementary Materials}

The Supplementary Materials include the following Figures and Tables: Figure SI-1. Workflow for the ddMS ${ }^{3}$ used in the AcquireX Deep Scan mode. Figure SI-2. Workflow for the $\mathrm{ddMS}^{3}$ used in the AcquireX IPE mode. Figure SI-3. Compound discover workflow. Figure SI-4. Log P and retention time (Rt) of the quality control compounds. Table SI1. Description of the different nodes of the CD workflow. Table SI-2. Calculation of the average response factor. Table SI-3. Experimental $\mathrm{MS}^{3}$ transitions. (Supplementary Materials)

\section{References}

[1] EU, 2011. COMMISSION REGULATION (EU) No 10/2011 of 14 January 2011 on plastic materials and articles intended to come into contact with food.

[2] EFSA, "Panel on food contact materials, enzymes, flavourings and processing aids," EFSA Journal, vol. 14, no. 1, p. 4357, 2016.

[3] ILSI Europe, Guidance on Best Practices on the Risk Assessment of Non Intentionally Added Substances (NIAS) in Food Contact Materials and Articles, 2015, ISBN: 9789078637424.

[4] EU, 2004, Regulation (EC) No 1935/2004 of the European Parliament and of the Council of 27 October 2004 on materials and articles intended to come into contact with Food and Repealing Directives 80/590/EEC and 89/109/EEC.

[5] K. Bhunia, S. S. Sablani, J. Tang, and B. Rasco, "Migration of chemical compounds from packaging polymers during microwave, conventional heat treatment, and storage," Comprehensive Reviews in Food Science and Food Safety, vol. 12, no. 5, pp. 523-545, 2013.

[6] C. Bignardi, A. Cavazza, C. Laganà, P. Salvadeo, and C. Corradini, "UHPLC-high-resolution mass spectrometry determination of bisphenol $\mathrm{A}$ and plastic additives released by polycarbonate tableware: influence of ageing and surface damage," Analytical and Bioanalytical Chemistry, vol. 407, no. 26, pp. 7917-7924, 2015.

[7] E. J. Hoekstra and C. Simoneau, "Release of bisphenol A from polycarbonate-A review," Critical Reviews in Food Science and Nutrition, vol. 53, no. 4, pp. 386-402, 2013.

[8] Y. Zhang, J. Li, and Y. Yan, "Molecular dynamics study of the migration of bisphenol A from polycarbonate into food simulants," Chemical Physics Letters, vol. 741, Article ID 137125, 2020.
[9] Y. Sanchis, V. Yusà, and C. Coscollà, "Analytical strategies for organic food packaging contaminants," Journal of Chromatography A, vol. 1490, pp. 22-46, 2017.

[10] M. Calow, P. Alfaro, C. Nerín, E. Jones, and E. Riches, "Progress in mass spectrometry for the analysis of set-off phenomena in plastic food packaging materials," Journal of Chromatography A, vol. 1453, pp. 124-133, 2016.

[11] C. Nerin, P. Alfaro, M. Aznar, and C. Domeño, "The challenge of identifying non-intentionally added substances from food packaging materials: a review," Analytica Chimica Acta, vol. 775, pp. 14-24, 2013.

[12] M. J. Martínez-Bueno, M. J. Gómez Ramos, A. Bauer, and A. R. Fernández-Alba, "An overview of non-targeted screening strategies based on high resolution accurate mass spectrometry for the identification of migrants coming from plastic food packaging materials," TrAC Trends in Analytical Chemistry, vol. 110, pp. 191-203, 2019.

[13] C. Bignardi, A. Cavazza, C. Corradini, and P. Salvadeo, "Targeted and untargeted data-dependent experiments for characterization of polycarbonate food-contact plastics by ultra high performance chromatography coupled to quadrupole orbitrap tandem mass spectrometry," Journal of Chromatography a, vol. 1372, pp. 133-144, 2014.

[14] C. Bignardi, A. Cavazza, C. Laganà, P. Salvadeo, and C. Corradini, "Release of non-intentionally added substances (NIAS) from food contact polycarbonate: effect of ageing," Food Control, vol. 71, pp. 329-335, 2017.

[15] Thermo Scientific, "SN65392-EN 0119M. AcquireX," 2019, Available on themofisher.com/AcquireX. Accessed May 2020.

[16] Thermo Scientific, Thermo Scientific, 2019, Available on: https://www.thermofisher.com/es/es/home/industrial/massspectrometry/liquid-chromatography-mass-spectrometrylc-ms/lc-ms-software/multi-omics-data-analysis/compounddiscoverer-software.html.

[17] E. Canellas, P. Vera, and C. Nerín, "Migration assessment and the 'threshold of toxicological concern' applied to the safe design of an acrylic adhesive for food-contact laminates," Food Additives and Contaminants: Part A, vol. 34, no. 10, pp. 1721-1729, 2017.

[18] EFSA, "Scientific Opinion on Exploring options for providing advice about possible human health risks based on the concept of Threshold of Toxicological Concern (TTC)," EFSA Journal, vol. 10, no. 7, p. 2750, 2012.

[19] V. Yusà, A. López, P. Dualde et al., "Analysis of unknowns in recycled LDPE plastic by LC-Orbitrap Tribrid HRMS using MS3 with an intelligent data acquisition mode," Microchemical Journal, vol. 158, p. 105256, 2020.

[20] FCA, FCA Guidelines on Risk Assessment of Non-listed Substances (NLS) and Non-intentionally Added Substances (NIAS) under the Requirements of Article 3 of the Framework Regulation, EC) 1935/2004, 2016.

[21] ILE Europe, Guidance on Best Practices on the Risk Assessment of Non Intentionally Added Substances (NIAS) in Food Contact. Report Commissioned by the Packaging Materials Task Force, 2015.

[22] NHI, National Center of Advancing Translational Sciences, 2020, https://drugs.ncats.io/drug/95VTI93VUL.

[23] S. P. Bhatia, L. Jones, C. S. Letizia, and A. M. Api, "Fragrance material review on 4-tert-butylcyclohexyl acetate," Food and Chemical Toxicology, vol. 46, no. 12, pp. S36-S41, 2008.

[24] CosIng, European Commission Database for Information on Cosmetic Substances and Ingredients, Accessed on 2020, https:// ec.europa.eu/growth/sectors/cosmetics/cosing_en, 2020. 
[25] Z. Bodai, C. Kirchkeszner, M. Novák et al., "Migration of tinuvin $\mathrm{P}$ and irganox 3114 into milk and the corresponding authorised food simulant," Food Additives and Contaminants: Part A, vol. 32, no. 8, pp. 1358-1366, 2015.

[26] M. T. D. A. Freire, I. A. Santana, and F. G. R. Reyes, "Plasticizers in brazilian food-packaging materials acquired on the retail market," Food Additives and Contaminants, vol. 23, no. 1, pp. 93-99, 2006.

[27] EFSA, "Camphor in flavourings and other food ingredients with flavouring properties opinion of the scientific panel on food additives, flavourings, processing aids and materials in contact with food on a request from the commission," The EFSA Journal, vol. 729, pp. 1-15, 2008.

[28] H. B. Pyeon, J. E. Park, and D. H. Suh, "Non-phthalate plasticizer from camphor for flexible pvc with a wide range of available temperature," Polymer Testing, vol. 63, pp. 375-381, 2017.

[29] ECHA, Mapping Exercise. Plastic Additive Iniciative, 2020, https://echa.europa.eu/fr/mapping-exercise-plastic-additivesinitiative\#table. Acceded June 2020.

[30] M. Biedermann and K. Grob, "GC method for determining polyadipate plasticizers in foods: transesterification to dibutyl adipate, conversion to migrating polyadipate," Chromatographia, vol. 64, no. 9-10, pp. 543-552, 2006.

[31] Pubchem, Pubchem, 2020, https://pubchem.ncbi.nlm.nih. gov/. Accesed May 2020.

[32] Mass Frontier, Mass Frontier, 2020, https://www. thermofisher.com/es/es/home/industrial/mass-spectrometry/ liquid-chromatography-mass-spectrometry-lc-ms/lc-mssoftware/multi-omics-data-analysis/mass-frontier-spectralinterpretation-software.html.

[33] E. L. Schymanski, J. Jeon, R. Gulde et al., "Identifying small molecules via high resolution mass spectrometry: communicating confidence," Environmental Science \& Technology, vol. 48, no. 4, pp. 2097-2098, 2014.

[34] A. Shrivastava, Introduction to Plastic Engineering. PDL Handbook Series, Elsevier, New York, NY, USA, 2018.

[35] R. Jamarani, H. Erythropel, J. Nicell, R. Leask, and M. Marić, "How green is your plasticizer?" Polymers, vol. 10, no. 8, p. $834,2018$.

[36] REACH, "Authorisation list," 2020, https://echa.europa.eu/ es/home. Acceded Jun 2020.

[37] J. N. Hahladakis, C. A. Velis, R. Weber, E. Iacovidou, and P. Purnell, "An overview of chemical additives present in plastics: migration, release, fate and environmental impact during their use, disposal and recycling," Journal of Hazardous Materials, vol. 344, pp. 179-199, 2018.

[38] EU, Commission implementing Regulation (EU) No 872/ 2012 of 1 October 2012 adopting the list of flavouring substances provided for by Regulation (EC) No 2232/96 of the European Parliament and of the Council, introducing it in Annex I to Regulation (EC) No 1334/2008 of the European Parliament and of the Council and repealing Commission Regulation (EC) No 1565/2000 and Commission Decision 1999/217/EC, 2012.

[39] C. A. M. Eke, J. L. Marks, L. B. Wroblewski, H. S. Raatikainen, S. R. Lenox, and K. E. Gebhardt, "Human and environmental toxicity of sodium lauryl sulfate (SLS): evidence for safe use in household cleaning products," Environmental Health Insights, vol. 9, pp. EHI.S31765-32, 2015.

[40] M. A. da Silva, V. Calabrese, J. Schmitt et al., "Impact of wormlike micelles on nano and macroscopic structure of TEMPO-oxidized cellulose nanofibril hydrogels," Soft Matter, vol. 16, no. 20, pp. 4887-4896, 2020.
[41] R. Benson, "Hazard to the developing male reproductive system from cumulative exposure to phthalate esters-dibutyl phthalate, diisobutyl phthalate, butylbenzyl phthalate, diethylhexyl phthalate, dipentyl phthalate, and diisononyl phthalate," Regulatory Toxicology and Pharmacology, vol. 53, no. 2, pp. 90-101, 2009.

[42] V. García Ibarra, A. Rodríguez Bernaldo de Quirós, P. Paseiro Losada, and R. Sendón, "Non-target analysis of intentionally and non intentionally added substances from plastic packaging materials and their migration into food simulants," Food Packaging and Shelf Life, vol. 21, Article ID 100325, 2019.

[43] ECCS, "ECCS.Data bank," 2020, Available at: http://eccs. unicartagena.edu.co/data_sheet.php?id=131\&varurl=data. Accessed May 2020.

[44] Y. Yan, C.-Y. Hu, Z.-W. Wang, and Z.-W. Jiang, "Degradation of irgafos 168 and migration of its degradation products from PP-R composite films," Packaging Technology and Science, vol. 31, no. 10, pp. 679-688, 2018.

[45] A. M. Api, D. Belsito, S. Bhatia et al., "fragrance ingredient safety assessment, methyl hexyl oxo cyclopentanone carboxylate, CAS registry number 37172-53-5," Food and Chemical Toxicology, vol. 110, no. Suppl 1, pp. S327-S336, 2017.

[46] A. Olejnik, A. Sliwowska, and I. Nowak, "Jasmonic acid, methyl jasmonate and methyl dihydrojasmonate as active compounds of topical formulations," Colloids and Surfaces A: Physicochemical and Engineering Aspects, vol. 558, pp. 558569, 2018.

[47] EFSA, "Update of the risk assessment of di-butylphthalate (DBP), butyl-benzyl-phthalate (BBP), bis(2-ethylhexyl) phthalate (DEHP), di-isononylphthalate (DINP) and di-isodecylphthala te (DIDP) for use in food contact materials," EFSA Journal, vol. 17, no. 12, p. 5838, 2019.

[48] EPA, Phthalates TEACH Chemical Summary, 2020, https:// archive.epa.gov/region5/teach/web/pdf/phthalates_summary. pdf Accessed May 2020. 